\title{
Computer embedded system development at the core of technical analysis
}

\author{
Zenghui Zhao ${ }^{1, a}$ GuoJian Cheng $^{1, \text { a }}$ \\ ${ }^{1}$ XI’ AN PEIHUA UNIVERSITY, Xi' an 710125, China \\ Azzhsmseminer@126.com
}

Keywords: : embedded ; computer system ; core technology

Abstract.With the constant development and popularization of computer information technology, people for the dependence of the computer control system is also increasing, using computer to realize the control of other electronic equipment is the important trend of the current computer technology.Embedded system development and application of the auxiliary function of computer operating system, through the software and hardware to cooperate on other devices for automation control, enriches the model function of computer application technology, promotes the development of the computer system development and equipment operation control.The core of the computer embedded system in this paper, the development of related technology are analyzed.

\section{Introduction}

The computer's operating system can't through the execution of the program code to realize the direct control of the equipment and adjust, but in order to adapt to the people to the requirement of the computer system, must want to pay attention to the development of embedded system, regulation of auxiliary equipment is completed by computer operating system to promote the improvement of enterprise management automation level of production.System development personnel should according to the different requirements of users to adjust the function of the embedded system operation module, to promote intelligent computer system level and application efficiency[1].

\section{The characteristics of the embedded system}

(I). The performance-based characteristics

Application of the embedded system of computer operating system, an important change is reflected in the original function is relatively single operation of the system function increased significantly, the higher efficiency of execution of the program code complexity and processing effect is greatly increased.Unlike traditional computer operating system, embedded system structure is more complex, but its data processing model is more simple, is the original simple processing mode combination and collocation, with the interaction between different complex components and unified operating system data processing technology to the professional interpretation and processing of information, in order to ensure that fully realized of the embedded system function need to be in the original system components increase in the micro processor.Application of the embedded system to improve the automation level of the computer operation, through the system of internal data quality, raise the efficiency of the operation of the computer system.

(II) The personalized characteristic

This is the most outstanding feature of embedded system.In embedded system development and most widespread popularity before computer USES universal control system, the research and development of all the computer users oriented control system function and module of small differences, unable to meet the personalized needs of user groups.And through a customized embedded system and programmable integrated circuit and the application of professional processor technology effectively to improve the pertinence of computer operating system, the personalized needs of user groups can be met.Embedded system has realized the small space multifunctional 
module combination, efficient way of data processing and individual function module makes its application in the social production and People's Daily life increasingly widespread.

( III).Long-term characteristics

Compared with the traditional single program computer operating system, embedded system has a significant long-term characteristics.Full application of embedded system development of modernization, intelligent computer data information analysis and processing technology, computer users through a series of operation will be different types of data within the processing equipment of the computer system, make its become intelligent data code, the code into a command message to a device, the running state of the equipment according to instructions and other changes.Embedded system can be operated according to the different needs of users and real experience to optimize the processing pattern and improve the pertinence and more selective, that is the future of computer science and technology developing compete in the technology of embedded system and other significant advantages.Science and technology industry especially in the computer industry market development model transformation to a great extent, need to use embedded systems, and embedded systems in the market of the future has the huge development potential, which is its long-term performance.

( IV ).Integrated features

Comprehensive is another important feature of embedded computer system.Embedded systems need to be in the process of development and application of several technology to integrate and use science and technology, including electronic information technology, computer application technology, software development technology and wireless communication technology and so on.Comprehensive characteristics of this technology for embedded system development of science and technology personnel overall quality as well as research and development unit of capital investment put forward higher requirements, comprehensive performance and make the embedded system presents the scientific research, personnel allocation and intensive characteristics of scientific research funds. The comprehensive characteristics of embedded integrated system makes it can be used in a variety of functions of computer operating system.

\section{Embedded processor technology in the development of the system}

\section{(I). General technology}

Different processor mode of data processing, data processing type and there are significant differences in scope.General-purpose processor is the biggest advantage of its wide applicability and the feature of diversity, through the application of the general-purpose processor embedded system can be versatile.General-purpose processors can convert their stored procedures, to ensure that its in a different computer operating system can realize the safety storage.The application of the embedded computer system of general processor technology and operation, extend the applicability of the data transmission path, generic data transfer mode to improve processing efficiency of computer logic unit, on the one hand, on the other hand also can guarantee the normal operation of the register data[2].

(II) A single technology

A single processor technology marked characteristics embodied in the function of the oneness and targeted to a specific user group.In order to improve the security of information system and part of the user groups do not need to diversified operation function, in this context with a single processor system function relatively single technology application.Single processor can technology are often applied to a specific application is relatively narrow, but the function is more compact, the user can according to their own requirements of instructions issued and system receives the command and response speed is greatly increased.In the decoder of embedded computer system is applied to a single processor technology, this technology has the encoding and decoding of data transmission to the special features, the function of the application in the maximum extent to ensure the normal use of the computer system.Is helpful for people in life and work to the operation of the computer more convenient.

( III).Special technology 
Modern social division of labor is increasingly clear, people are shifting to the requirement of function of computer system, to the pertinence, specificity and reliability of the system function put forward higher requirements. The main purpose of computer embedded system development is optimize the traditional general-purpose processor technology, realizing the function of computer system application optimal Settings.Special processor technology according to the specific requirements of the equipment will be set as the system processor control components, information of the bearing is mainly digital signal, realize information through digital signal transmission channel of interaction and communication.Developers to the execution of the application function code stored in a dedicated processor chip, when the system can realize the issue related to the executing instructions direct operating computer embedded system.The important factors in the computer system of computer system and change needs to be applied in intelligent upgrade dedicated processor technology, accelerated the rapid development of the computer system.

\section{Integrated circuit technology in embedded system development}

\section{(I). All custom IC and VLSI}

The application of the integrated circuit technology has realized the computer embedded system XianLv connection of electronic components, to improve the conformity of the system components and user operation needs, the reliability of the computer system can be guaranteed.Full custom IC technology in the application process, designers need to embedded system based on the specific number, chip installation location, the size of the transistor, such as the map to different data layer and the area of the chip is optimized, thus effectively improving the speed of embedded system chip, energy and resources utilization.On a large scale integrated circuit design and production needs, in turn, IC design, and relying on the mask according to the design requirements for the production of chip production, it requires a lot of time cost and NRE cost, in the strict performance and functional diversity of embedded system is more suitable[3].

(II) Half a custom ASIC

Compared with all custom IC, semi custom ASIC design and application of the binding is stronger, standard unit design method and the gate array design method is half custom ASIC design method of two main application of embedded system application of the integrated circuit module and system structure design of matching the demand is higher.Half a custom ASIC USES control chip for universal unit element and element group, in the process design for circuit logic and the coordination between different functions and operating rationality to give enough attention.Half a custom ASIC design of integrated circuit application flexibility and higher operating more simple, the performance is relatively good, time can effectively control circuit design, ensure that the quality of the chips and computer embedded system development design cycle.

( III).Programmable ASIC

Programmable ASIC integrated circuit technology in the development of the current computer embedded system has wide application range, in the process of circuit design IC manufacturers don't have to participate in, but the editor's existing related circuit in each layer, the lab according to the specific requirements of the system can carry out chip production, embedded system development time can be effectively controlled.Besides programmable ASIC cheaper, but the unit power consumption is higher, and the data transmission and speed of the movement of the chip are disc.The programmable ASIC integrated circuit technology is mainly used in the development of advanced technology industry computer embedded system, improve the utilization ratio of the operating system.

\section{III.The development of embedded systems}

(I). The collection of hardware

System developers need to clear the focus of the embedded system development, the spell of simple model and system upgrade myth, improve for hardware acquisition value, as the system 
development mainly focus on technology, the hardware equipment, the overall optimization, ensure the realization of the function of the embedded system integrated.Researchers need to look at the system, with the idea of the whole circuit board design and chip level are determined as the highest configuration, so as to realize the intelligent processing of system data, to ensure that the embedded system in different areas and module function under the combination can be normal use, and for the development of computer technology in the future to lay the good foundation.

(II)Pay attention to the coordination between different software

Embedded systems development and utilization of the main purpose is to improve performance of the operation of the computer and the control of the other electronic equipment efficiency, in order to ensure the normal operation of the embedded system, it is important to cooperate with each other between different software, in the process of system development researchers to attach importance to the combination between software and function of sex.Current social users the use of computers has been basically can realize the intelligent selection and analysis of the data, so as to improve the accuracy of the command execution.Computer embedded system through the integration of system function, realize the hierarchical data processing and the effectiveness and efficiency of data processing is also important for embedded system for the assessment of indicators, the essence of embedded system application is the same hardware equipment under the environment to realize the integration of different functions, and the match is to realize the function of software integration of the necessary conditions[4].

( III).The design of the background support system

In order to ensure the normal use of embedded computer system need to attach importance to the background support system design, system operation and data processing to provide a solid hardware support.Computer language only programmers can read, so the primary purpose of system development is the conversion between natural language and computer language.People in order to prevent computer language program hinder the process of system development, need to be in the process of program language to write the discrimination and classification of language.Embedded system put into use before need to inspect program function, to ensure the smooth implementation of multilingual standard and accurate launch system.

\section{ACKNOWLEDGEMENTS}

To sum up, the development and application of the embedded system by changing the enterprise equipment control mode to improve the enterprise management efficiency and profitability.In the future development of enterprises will continue to increase investment in research and development of embedded system, through the processor and the application of the integrated circuit technology to reduce the difficulty of computer programming language to write, to improve the effectiveness of the application of embedded system, improve cooperation degree of computer hardware and software, improve the application practice of computer system environment, to ensure the smooth realization of the function of computer application.

\section{References:}

[1]Xiong Xuhui For the Internet of things engineering for the development of embedded system experimental design [J] - journal of hubei normal university (natural science edition), 2016 (01) [2] hai-cheng liu ability structure of embedded development engineers and cultivation research [J] 2016 single chip microcomputer and embedded system application (08)

[3]FanHongWei rotor on-line dynamic balancing embedded control system development and validation [J] - vibration, 2015 (04) test and diagnosis

[4] Liu Xiang .computer remote main technical points of the embedded system development to explore communication [J] - 2016 (04) in the world 